\title{
A novel self-tuning fractional order PID control based on optimal model reference adaptive system
}

\author{
Mohamed. A. Shamseldin', Mohamed Sallam², A. M. Bassiuny ${ }^{3}$, A. M. Abdel Ghany ${ }^{4}$ \\ ${ }^{1}$ Department of Mechatronics Engineering, Future University in Egypt, Cairo, Egypt \\ ${ }^{2,3}$ Department of Mechanical Engineering, Helwan University, Cairo, Egypt \\ ${ }^{4}$ Department of Electrical Engineering, October 6 University (Helwan University Originally), Cairo, Egypt
}

\begin{tabular}{l}
\hline \hline Article Info \\
\hline Article history: \\
Received Jul 12, 2018 \\
Revised Nov 19, 2018 \\
Accepted Dec 13, 2018 \\
\hline
\end{tabular}

Keywords:

FOPID control Harmony research (HS) Model reference

\begin{abstract}
This paper presents a novel self-tuning fractional order PID (FOPID) control based on optimal Model Reference Adaptive Control (MRAC). The proposed control technique has subjected to a third order system case study (power system load frequency control). The model reference describes the requirements of designer. It can be first or second order system. The parameters of MRAC have obtained using the harmony search (HS) optimization technique to achieve the optimal performance. Sometimes, the tuning of the five parameters of FOPID control online at same moment consumes more calculation time and more processing. So, this study proposes three methods for self-tuning FOPID control. The first method has been implemented to tune the two integral and derivative parameters only and the rest of parameters are fixed. The second method has been designed to adjust the proportional, integral derivative parameters while the other fractional parameters are constant. The last method has developed to adjust the five parameters of FOPID control simultaneously. The simulation results illustrate that the third method of self-tuning FOPID control can accommodate the sudden disturbance compared to other techniques. Also, it can absorb the system uncertainty better than the other control techniques.
\end{abstract}

Copyright (c) 2019 Institute of Advanced Engineering and Science. All rights reserved.

\section{Corresponding Author:}

A. M. Abdel Ghany,

Department of Electrical Engineering,

October 6 University (Helwan University Originally), Cairo, Egypt.

Email: ghanyghany@hotmail.com

\section{INTRODUCTION}

The PID control has been applied to process control in most of engineering applications for decades [1]. The PID control has simple structure and linear behavior. Also, it gives acceptable performance for several industrial applications [2]. There are several methods to select the proper values for PID controller parameters [3]. The traditional methods for selecting these parameters such as try and error and ZieglerNichols which were became inappropriate to achieve a good performance [4]. So, the researchers have tended to use alternative methods such as optimization techniques (Genetic Algorithm (GA), Particle Swarm Optimization (PSO), Ant Colony Optimization (ACO) and Harmony Search (HS)) which are trying to reach the optimal solution for controller parameters [5]. Still, the behavior of PID control is linear and cannot deal with the high disturbance and high nonlinearity of complicated systems [6], [7].

The fractional order PID (FOPID) control has been widely used in control engineering in recent decades [8]. The FOPID considers the nonlinear copy of PID control where two more parameters (the fractional integral and derivative) added to the PID control parameters [9]. Hence, the task of designer selecting the proper values for the five parameters of the FOPID control [10]. The FOPID control can solve the nonlinearity problem but it cannot deal with the sudden disturbance due to its parameters which still fixed [11]. 
Different techniques have been proposed to tune the five parameters of FOPID control online but most of these techniques are based on the fuzzy logic control [12], [13]. The fuzzy logic control can solve the uncertainty problem and sudden disturbance but its design depends on the experience which sometimes is not available for some systems [14]-[16]. This study presents a novel technique to tune the FOPID control parameters online based on optimal model reference adaptive control (MRAC). It is known that the MRAC is high ranking adaptive control where it forces the overall system to follow the behavior of preselected model reference [17]. The preselected model can be first or second order system according to the point of view the designer and complicated degree of the system [18].

The task of model reference adaptive control is adjusting the FOPID control parameters online. The model reference contains the desired performance which can satisfy the designer. Moreover, to guarantee high performance the parameters of model reference optimized using the harmony search (HS) optimization technique according to a certain cost function.

The proposed technique will be subjected to a third order system as case study (power system load frequency control). Also, the proposed technique has been implemented with different methods. The first method has been designed to the MRAC will tune the two integral and derivative parameters only and the rest of parameters are fixed. The second method has been developed to adjust the proportional, integral derivative parameters while the other fractional parameters are constant. The last method fabricated to adjust the five parameters of FOPID control at the same time. The paper has organized as follows, firstly, the system model is presented. Secondly, the proposed control techniques are demonstrated. Thirdly, the simulation results are illustrated. Finally the conclusion is discussed.

\section{THIRD ORDER CASE STUDY MODEL}

Several nonlinear models for large power systems has to be established but all are complicated models. So, the linearized model has been used in our work. The power system represented by an equivalent turbine, governor and generator system [19], [20]. The transfer function of a single area power system model in a closed loop form as shown in Figure 1.

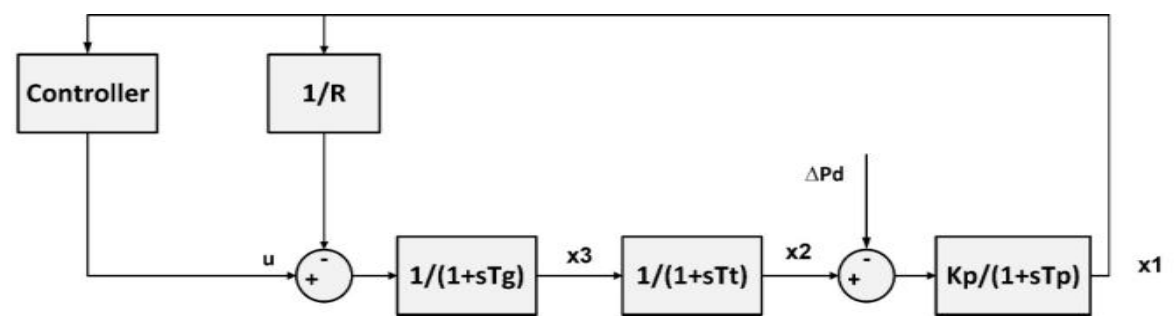

Figure 1. Block diagram of a single area power system

Where is $\mathrm{u}$ the control action, $\mathrm{d}$ is the disturbance vector $(\Delta \mathrm{Pd}), \mathrm{Tp}$ is the plant model time constant, $\mathrm{Tt}$ is the turbine time constant, $\mathrm{Tg}$ is the governor time constant, $\mathrm{Kp}$ is the plant gain, $\mathrm{R}$ is the speed regulation due to governor action, $\mathrm{x} 1$ is the change in system frequency, $\mathrm{x} 2$ is the incremental changes in generator output and $\mathrm{x} 3$ is the governor valve position. Table 1 present the values of system parameters that used in this work.

\begin{tabular}{|c|c|}
\hline Parameters & Value \\
\hline $\mathrm{Kp}$ & $120 \mathrm{pu}$ \\
\hline $\mathrm{Tp}$ & $20 \mathrm{~s}$ \\
\hline $\mathrm{Tt}$ & $0.3 \mathrm{~s}$ \\
\hline $\mathrm{Tg}$ & $0.08 \mathrm{~s}$ \\
\hline $\mathrm{R}$ & $2.4 \mathrm{~Hz} / \mathrm{pu} . \mathrm{MW}$ \\
\hline
\end{tabular}

\section{SELF-TUNING FOPID CONTROL}

The Model Reference Adaptive Control (MRAC) is high-ranking adaptive controller [2], [5]. It may be regarded as an adaptive servo system in which the desired performance is expressed in terms of a

A novel self-tuning fractional order PID control based on optimal model... (Mohamed. A. Shamseldin) 
reference model. In this work the FOPID control parameters will be adjusted on-line using the model reference technique as shown in Figure 2.

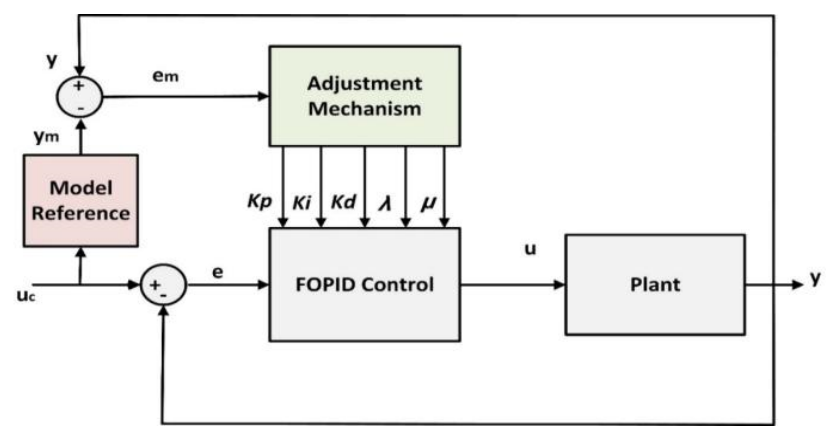

Figure 2. The overall system with self-tuning FOPID based on model reference technique

To adjust parameters using MIT rule which use the following loss function

$$
j(\underline{\theta})=\frac{1}{2} e_{m}^{2}
$$
$\mathrm{j}$, that is,

To make $\mathrm{j}$ small, it is reasonable to change the parameters in the direction of the negative gradient of

$$
\frac{d \underline{\theta}}{d t}=-\gamma \frac{\partial j}{\partial \underline{\theta}}=-\gamma e_{m} \frac{\partial e_{m}}{\partial \underline{\theta}}
$$

where $\gamma$ stand for the adaptation gain while $\underline{\theta}$ is the centroid vector of the output membership function. The transfer function of FOPID control can be described as follows.

$$
\begin{aligned}
& \frac{u(s)}{e(s)}=k_{p}+k_{i} \frac{1}{s^{\lambda}}+k_{d} s^{\mu} \\
& e=u_{c}-y
\end{aligned}
$$

Assume that the plant can be simplified to a first order system as obvious in the following (5).

$$
\frac{y(s)}{u(s)}=\frac{k}{T s+1}
$$

where $k$ and $T$ are unknown parameters. Also, assume that the model reference takes a form first order system as the following relationship.

$$
\frac{y_{m}(s)}{u_{c}(s)}=\frac{k_{m}}{T_{m} s+1}
$$

Where $k_{m}$ and $T_{m}$ are selected by designer.From (4-6) can conclude that

$$
\begin{aligned}
& y=\frac{k}{T s+1}\left(k_{p}+k_{i} \frac{1}{s^{\lambda}}+k_{d} s^{\mu}\right)\left(u_{c}-y\right) \\
& \stackrel{\text { yields }}{\longrightarrow} \quad y=\frac{k k_{p}+k k_{i} \frac{1}{s^{\lambda}}+k k_{d} s^{\mu}}{T s+1} u_{c}-\frac{k k_{p}+k k_{i} \frac{1}{s^{\lambda}}+k k_{d} s^{\mu}}{T s+1} y
\end{aligned}
$$




$$
\begin{aligned}
& \left(1+\frac{k k_{p}+k k_{i} \cdot \frac{1}{s^{\lambda}}+k k_{d} s^{\mu}}{T s+1}\right) y=\frac{k k_{p}+k k_{i} \cdot \frac{1}{s^{\lambda}}+k k_{d} s^{\mu}}{T s+1} u_{c} \\
& \left(\frac{T s+1+k k_{p}+k k_{i} \cdot \frac{1}{{ }^{\lambda} \lambda}+k k_{d} s^{\mu}}{T s+1}\right) y=\frac{k k_{p}+k k_{i} \cdot \frac{1}{{ }^{2} \lambda}+k k_{d} s^{\mu}}{T s+1} u_{c} \\
& y=\frac{k k_{p}+k k_{i} \cdot \frac{1}{{ }^{\lambda} \lambda}+k k_{d} s^{\mu}}{T s+1+k k_{p}+k k_{i} \cdot \frac{1}{{ }^{\lambda}}+k k_{d} s^{\mu}} u_{c} \\
& e_{m}=y-y_{m} \\
& e_{m}=\left[\frac{k k_{p}+k k_{i} \cdot \frac{1}{s} \lambda+k k_{d} s^{\mu}}{T s+1+k k_{p}+k k_{i} \cdot \frac{1}{{ }^{\lambda}}+k k_{d} s^{\mu}}-\frac{k_{m}}{T_{m} s+1}\right] u_{c}
\end{aligned}
$$

\subsection{Adaptation law of $\boldsymbol{k}_{\boldsymbol{p}}$ parameter}

This sub-section shows the steps of design of the adaptation law for proportional gain parameter $\left(k_{p}\right)$. By deriving (10) respect to the proportional gain $\left(k_{p}\right)$ to obtain the following relationship.

$\frac{\partial e_{m}}{\partial k_{p}}=\left[\frac{k}{T s+k k_{p}+k k_{d} s^{\mu}+k k_{i} \cdot \frac{1}{s^{\lambda}}+1}-\frac{k\left(k k_{p}+k k_{i} \cdot \frac{1}{s^{\lambda}}+k k_{d} s^{\mu}\right)}{\left(T s+k k_{p}+k k_{d} s^{\mu}+k k_{i} \cdot \frac{1}{s}+1\right)^{2}}\right] u_{c}$

(11) can be rewritten;

$$
\begin{aligned}
& \frac{\partial e_{m}}{\partial k_{p}}=\left[\frac{k\left(T s+k k_{p}+k k_{i} \cdot \frac{1}{s} \lambda+k k_{d} s^{\mu}+1-k k_{p}-k k_{i} \cdot \frac{1}{\lambda^{\lambda}}-k k_{d} s^{\mu}\right)}{\left(T s+k k_{p}+k k_{i} \cdot \frac{1}{s} \lambda+k k_{d} s^{\mu}+1\right)^{2}}\right] u_{c} \\
& \frac{\partial e_{m}}{\partial k_{p}}=\left[\frac{k(T s+1)}{\left(T s+k k_{p}+k k_{i} \cdot \frac{1}{s}+k k_{d} s^{\mu}+1\right)^{2}}\right] u_{c} \\
& \frac{\partial e_{m}}{\partial k_{p}}=\left[\frac{k(T s+1)}{\left(T s+k k_{p}+k k_{i} \cdot \frac{1}{s}+k k_{d} s^{\mu}+1\right)\left(k k_{p}+k k_{i} \cdot \frac{1}{s}+k k_{d} s^{\mu}\right)}\right] y
\end{aligned}
$$

From (12) and (14);

$$
\frac{\partial e_{m}}{\partial k_{p}}=\left[\frac{k^{2} e}{\left(T s+k k_{p}+k k_{i} \cdot \frac{1}{s^{\lambda}}+k k_{d} s^{\mu}+1\right)}\right]
$$

To achieve the desired performance, the following condition must be hold.

$$
\begin{aligned}
& T s+k k_{p}+k k_{i} \cdot \frac{1}{s^{\lambda}}+k k_{d} s^{\mu}+1=T_{m} s+1 \\
& \frac{\partial e_{m}}{\partial k_{p}}=\frac{k^{2} e}{T_{m} s+1}
\end{aligned}
$$

From the MIT rule can obtain the following relationship

$$
\frac{d k_{p}}{d t}=-\gamma \cdot e_{m} \cdot \frac{k^{2} e}{T_{m} s+1}
$$




$$
\begin{aligned}
& \frac{d k_{p}}{d t}=-\gamma_{1} \cdot \frac{e_{m} \cdot e}{T_{m} s+1} \\
& \gamma_{1}=\gamma \cdot k^{2} \\
& \left.k_{p}\right)_{\text {new }}=\int \frac{d k_{p}}{d t} d t+k_{p}(0)
\end{aligned}
$$

Where $k_{p}(0)$ is the initial value of proportional gain $k_{p}$.

\subsection{Adaptation law of $\boldsymbol{k}_{\boldsymbol{i}}$ parameter}

This sub-section shows the steps of design of the adaptation law for integral gain parameter $\left(k_{i}\right)$. By deriving (8) respect to the integral gain $\left(k_{i}\right)$ to obtain the following relationship.

$$
\frac{\partial e_{m}}{\partial k_{i}}=\frac{1}{s^{\lambda}}\left[\frac{k}{T s+k k_{p}+k k_{d} s^{\mu}+k k_{i} \cdot \frac{1}{s}+1}-\frac{k\left(k k_{p}+k k_{i} \cdot \frac{1}{s^{\lambda}}+k k_{d} s^{\mu}\right)}{\left(T s+k k_{p}+k k_{d} s^{\mu}+k k_{i} \cdot \frac{1}{s}+1\right)^{2}}\right] u_{c}
$$

From (22) can be rewritten;

$$
\begin{aligned}
& \frac{\partial e_{m}}{\partial k_{i}}=\frac{1}{s^{\lambda}}\left[\frac{k\left(T s+k k_{p}+k k_{i} \cdot \frac{1}{s^{\lambda}}+k k_{d} s^{\mu}+1-k k_{p}-k k_{i} \cdot \frac{1}{{ }^{\lambda}}-k k_{d} s^{\mu}\right)}{\left(T s+k k_{p}+k k_{i} \cdot \frac{1}{s^{\lambda}}+k k_{d} s^{\mu}+1\right)^{2}}\right] u_{c} \\
& \frac{\partial e_{m}}{\partial k_{i}}=\frac{1}{s^{\lambda}}\left[\frac{k(T s+1)}{\left(T s+k k_{p}+k k_{i} \cdot \frac{1}{s}+k k_{d} s^{\mu}+1\right)^{2}}\right] u_{c} \\
& \frac{\partial e_{m}}{\partial k_{i}}=\frac{1}{s^{\lambda}}\left[\frac{k(T s+1)}{\left(T s+k k_{p}+k k_{i} \cdot \frac{1}{s}+k k_{d} s^{\mu}+1\right)\left(k k_{p}+k k_{i} \cdot \frac{1}{s^{\lambda}}+k k_{d} s^{\mu}\right)}\right] y
\end{aligned}
$$

From (23) and (25);

$\frac{\partial e_{m}}{\partial k_{i}}=\frac{1}{s^{\lambda}}\left[\frac{k^{2} e}{\left(T s+k k_{p}+k k_{i} \cdot \frac{1}{s^{\lambda}}+k k_{d} s^{\mu}+1\right)}\right]$

To achieve the desired performance, the condition must be hold in (16).

$\frac{\partial e_{m}}{\partial k_{i}}=\frac{1}{s^{\lambda}} \frac{k^{2} e}{T_{m} s+1}$

From the MIT rule can obtain the following relationship

$$
\begin{aligned}
& \frac{d k_{i}}{d t}=-\gamma \cdot e_{m} \cdot \frac{1}{s^{\lambda}} \frac{k^{2} e}{T_{m} s+1} \\
& \frac{d k_{i}}{d t}=-\gamma_{2} \cdot \frac{e_{m} \cdot e}{T_{m} s+1} \\
& \gamma_{2}=\gamma k^{2} \cdot \frac{1}{s^{\lambda(0)}}=\gamma_{1} \frac{1}{s^{\lambda(0)}} \\
& \left.k_{i}\right)_{\text {new }}=\int \frac{d k_{i}}{d t} d t+k_{i}(0)
\end{aligned}
$$

Where $k_{i}(0)$ is the initial value of proportional gain $k_{i}$. 


\subsection{Adaptation Law of $\boldsymbol{k}_{d}$ Parameter}

This sub-section illustrates the steps of design of the adaptation law for derivative gain parameter $\left(k_{d}\right)$. By deriving (10) respect to the derivative gain $\left(k_{d}\right)$ to obtain the following relationship.

$$
\begin{aligned}
& \frac{\partial e_{m}}{\partial k_{d}}=\left[\frac{k s^{\mu}}{T s+k k_{p}+k k_{i} \frac{1}{s^{\lambda}}+k k_{d} s^{\mu}+1}-\frac{k s^{\mu}\left(k k_{p}+k k_{i} \frac{1}{s^{\lambda}}+k k_{d} s^{\mu}\right)}{\left(T s+k k_{p}+k k_{i} \frac{1}{s^{\lambda}}+k k_{d} s^{\mu}+1\right)^{2}}\right] u_{c} \\
& \frac{\partial e_{m}}{\partial k_{d}}=\left[\frac{k s^{\mu}\left(T s+k k_{p}+k k_{i} \frac{1}{s^{\lambda}}+k k_{d} s^{\mu}+1-k k_{p}-k k_{i} \frac{1}{s^{\lambda}}-k k_{d} s^{\mu}\right)}{\left(T s+k k_{p}+k k_{i} \frac{1}{s^{\lambda}}+k k_{d} s^{\mu}+1\right)^{2}}\right] u_{c} \\
& \frac{\partial e_{m}}{\partial k_{d}}=\left[\frac{k s^{\mu}(T s+1)}{\left(T s+k k_{p}+k k_{i} \frac{1}{s^{\lambda}}+k k_{d} s^{\mu}+1\right)^{2}}\right] u_{c} \\
& \frac{\partial e_{m}}{\partial k_{d}}=\left[\frac{k s^{\mu}(T s+1)}{\left(T s+k k_{p}+k k_{i} \frac{1}{s^{\lambda}}+k k_{d} s^{\mu}+1\right)\left(k k_{p}+k k_{i} \frac{1}{s^{\lambda}}+k k_{d} s^{\mu}\right)}\right] y
\end{aligned}
$$

Also, from (33) and (35)

$$
\begin{aligned}
& \frac{\partial e_{m}}{\partial k_{d}}=\left[\frac{k^{2} \cdot s^{\mu} \cdot e}{\left(T s+k k_{p}+k k_{i} \frac{1}{s^{\lambda}}+k k_{d} s^{\mu}+1\right)}\right] \\
& \frac{\partial e_{m}}{\partial k_{d}}=\frac{k^{2} \cdot s^{\mu} \cdot e}{T_{m} s+1} \\
& \frac{d k_{d}}{d t}=-\gamma \cdot e_{m \cdot \frac{k^{2} \cdot s^{\mu} \cdot e}{T_{m} s+1}} \\
& \frac{d k_{d}}{d t}=-\gamma_{3} \cdot \frac{e_{m} \cdot e}{T_{m} s+1} \\
& \gamma_{3}=\gamma \cdot k^{2} \cdot s^{\mu(0)}=\gamma_{1} \cdot s^{\mu(0)} \\
& \left.k_{d}\right)_{n e w}=\int \frac{d k_{d}}{d t} d t+k_{d}(0)
\end{aligned}
$$

Where $k_{d}(0)$ is the initial value of derivative gain $k_{d}$.

\subsection{Adaptation Law of $\lambda$ Parameter}

This sub-section illustrates the steps of design of the adaptation law for fractional integral gain parameter $(\lambda)$. By deriving (10) respect to the fractional integral gain $(\lambda)$ to obtain the following relationship.

$$
\begin{aligned}
& \frac{\partial e_{m}}{\partial \lambda}=\frac{k k_{i} \ln (s)}{s^{\lambda}}\left[\frac{\left(k k_{p}+k k_{i} \frac{1}{s^{\lambda}}+k k_{d} s^{\mu}\right)}{\left(T s+k k_{p}+k k_{i} \frac{1}{s^{\lambda}}+k k_{d} s^{\mu}+1\right)^{2}}-\frac{1}{\left(T s+k k_{p}+k k_{i} \frac{1}{s^{\lambda}}+k k_{d} s^{\mu}+1\right)}\right] u_{c} \\
& \frac{\partial e_{m}}{\partial \lambda}=\frac{k k_{i} \ln (s)}{s^{\lambda}}\left[\frac{k k_{p}+k k_{i} \frac{1}{s^{\lambda}}+k k_{d} s^{\mu}-T s-k k_{p}-k k_{i} \frac{1}{s \lambda}-k k_{d} s^{\mu}-1}{\left(T s+k k_{p}+k k_{i} \frac{1}{s^{\lambda}}+k k_{d} s^{\mu}+1\right)^{2}}\right] u_{c} \\
& \frac{\partial e_{m}}{\partial \lambda}=\frac{k k_{i} \ln (s)}{s^{\lambda}}\left[\frac{-(T s+1)}{\left(T s+k k_{p}+k k_{i} \frac{1}{s^{\lambda}}+k k_{d} s^{\mu}+1\right)^{2}}\right] u_{c}
\end{aligned}
$$


$\frac{\partial e_{m}}{\partial \lambda}=\frac{k k_{i} \ln (s)}{s^{\lambda}}\left[\frac{-(T s+1)}{\left(T s+k k_{p}+k k_{i} \frac{1}{s^{\lambda}}+k k_{d} s^{\mu}+1\right)\left(k k_{p}+k k_{i} \frac{1}{s^{\lambda}}+k k_{d} s^{\mu}\right)}\right] y$

Also, from (41) and (43);

$$
\begin{aligned}
& \frac{\partial e_{m}}{\partial \lambda}=-\frac{k^{2} k_{i} \ln (s)}{s^{\lambda}}\left[\frac{e}{\left(T s+k k_{p}+k k_{i} \frac{1}{s^{\lambda}}+k k_{d} s^{\mu}+1\right)}\right] \\
& \frac{\partial e_{m}}{\partial \lambda}=-\frac{k^{2} k_{i} \ln (s)}{s^{\lambda}} \cdot \frac{e}{T_{m} s+1} \\
& \frac{d \lambda}{d t}=\gamma \cdot e_{m} \cdot \frac{k^{2} k_{i} \ln (s)}{s^{\lambda}} \cdot \frac{e}{T_{m} s+1} \\
& \frac{d \lambda}{d t}=\gamma_{4} \cdot \frac{e_{m} \cdot e}{T_{m} s+1} \\
& \gamma_{4}=\gamma \cdot \frac{k^{2} k_{i}(0) \ln (s)}{s^{\lambda(0)}}=\gamma_{2} \cdot k_{i}(0) \cdot \ln (s) \\
& \lambda)_{n e w}=\int \frac{d \lambda}{d t} d t+\lambda(0)
\end{aligned}
$$

Where $\lambda(0)$ is the initial value of fractional integral gain $\lambda$.

\subsection{Adaptation law of $\boldsymbol{\mu}$ parameter}

This sub-section demonstrates the steps of design of the adaptation law for fractional derivative gain parameter $(\mu)$. By deriving the (8) respect to the fractional derivative gain $(\mu)$ to obtain the following relationship.

$$
\begin{aligned}
& \frac{\partial e_{m}}{\partial \mu}=\left[\frac{k k_{d} \cdot s^{\mu} \cdot \ln (s)}{T s+k k_{p}+k k_{i} \frac{1}{s}+k k_{d} s^{\mu}+1}-\frac{k k_{d} \cdot s^{\mu} \cdot \ln (s)\left(k k_{p}+k k_{i} \frac{1}{s^{\lambda}}+k k_{d} s^{\mu}\right)}{\left(T s+k k_{p}+k k_{i} \frac{1}{s^{\lambda}}+k k_{d} s^{\mu}+1\right)^{2}}\right] u_{c} \\
& \frac{\partial e_{m}}{\partial \mu}=\left[\frac{k k_{d} \cdot s^{\mu} \cdot \ln (s)\left(T s+k k_{p}+k k_{i} \frac{1}{s^{\lambda}}+k k_{d} s^{\mu}+1-k k_{p}-k k_{i} \frac{1}{s^{\lambda}}-k k_{d} s^{\mu}\right)}{\left(T s+k k_{p}+k k_{i} \frac{1}{s^{\lambda}}+k k_{d} s^{\mu}+1\right)^{2}}\right] u_{c} \\
& \frac{\partial e_{m}}{\partial \mu}=\left[\frac{\left.k k_{d \cdot s^{\mu} \cdot \ln (s)(T s+1)}^{\left(T s+k k_{p}+k k_{i} \frac{1}{s^{\lambda}}+k k_{d} s^{\mu}+1\right)^{2}}\right] u_{c}}{\frac{\partial e_{m}}{\partial \mu}}=\left[\frac{k k_{d} \cdot s^{\mu} \cdot \ln (s)(T s+1)}{\left(T s+k k_{p}+k k_{i} \frac{1}{s^{\lambda}}+k k_{d} s^{\mu}+1\right)\left(k k_{p}+k k_{i} \frac{1}{\left.s^{\lambda}+k k_{d} s^{\mu}\right)}\right] y}\right.\right.
\end{aligned}
$$

Also, from (51) and (53);

$$
\begin{aligned}
& \frac{\partial e_{m}}{\partial \mu}=\left[\frac{k^{2} k_{d \cdot s^{\mu}} \cdot \ln (s) \cdot e}{\left(T s+k k_{p}+k k_{i} \frac{1}{s}{ }^{\lambda}+k k_{d} s^{\mu}+1\right)}\right] \\
& \frac{\partial e_{m}}{\partial \mu}=\frac{k^{2} k_{d \cdot s^{\mu} \cdot \ln (s) \cdot e}}{T_{m} s+1} \\
& \frac{d \mu}{d t}=-\gamma \cdot e_{m} \cdot \frac{k^{2} k_{d \cdot s^{\mu} \cdot \ln (s) \cdot e}}{T_{m} s+1}
\end{aligned}
$$




$$
\begin{aligned}
& \frac{d \mu}{d t}=-\gamma_{5} \cdot \frac{e_{m} \cdot e}{T_{m} s+1} \\
& \gamma_{5}=\gamma \cdot k^{2} \cdot k_{d}(0) \cdot s^{\mu(0)} \cdot \ln (s)=\gamma_{3} \cdot k_{d}(0) \cdot \ln (s) \\
& \mu)_{n e w}=\int \frac{d \mu}{d t} d t+\mu(0)
\end{aligned}
$$

Where $\mu(0)$ is the initial value of fractional integral gain $\mu$.

\subsection{Harmony search (HS) optimization}

The harmony search has been proposed in 2001. It is used lately in several engineering applications to obtain the optimal values of control parameters. In this study, the HS will be used to obtain the optimal parameters values of model reference adaptive control. The most effective parameter of MRAC is the adaptation gain for each adaptation law. Here, the harmony search tuning system will adjust the parameters of model reference adaptive system $\left(\gamma_{1}, \gamma_{2}, \gamma_{3}, \gamma_{4}\right.$ and $\left.\gamma_{5}\right)$ according to the objective function as shown in (60).

$$
f=\frac{1}{\left(1-e^{-\beta}\right)\left(M_{p}+e_{s S}\right)+e^{-\beta}\left(t_{s}-t_{r}\right)}
$$

Where $M_{p}$ the overshoot of system response is, $e_{s S}$ is the steady state error, $t_{s}$ is the settling time and $t_{r}$ is the rise time. Also, this objective function is able to compromise the designer demand by the weighting parameter value $(\beta)$. The parameter is set larger than 0.7 to reduce over shoot and steady-state error. If this parameter is set smaller than 0.7 the rise time and settling time will be reduced.

The initial population of Harmony Memory (HM) is produced randomly. HM contains Harmony Memory Solution (HMS) vectors. The HM is filled with HMS vectors as follows:

$$
H M=\left[\begin{array}{ccccc}
\gamma_{1_{(1,1)}} & \gamma_{2_{(1,2)}} & \gamma_{3_{(1,3)}} & \gamma_{4_{(1,4)}} & \gamma_{5_{(1,5)}} \\
\gamma_{1_{(2,1)}} & \gamma_{2_{(2,2)}} & \gamma_{3_{(2,3)}} & \gamma_{4_{(2,4)}} & \gamma_{5_{(2,5)}} \\
\cdot & \cdot & \cdot & \cdot & \cdot \\
\cdot & \cdot & \cdot & \cdot & \cdot \\
\cdot & \cdot & \cdot & \cdot & \cdot \\
\gamma_{1_{(H M S, 1)}} \gamma_{2} & \cdot & \cdot \\
(H M S, 2) & \gamma_{3(H M S, 3)} & \gamma_{4} \\
(H M S, 4) & \gamma_{5(H M S, 5)}
\end{array}\right]
$$

Table 2 demonstrates the obtained values of MRAC parameters after the offline tuning using the harmony search tuning system.

Table 2. MRAC Parameters

\begin{tabular}{cccccc}
\hline MRAC & $\gamma_{1}$ & $\gamma_{2}$ & $\gamma_{3}$ & $\gamma_{4}$ & $\gamma_{5}$ \\
\cline { 2 - 6 } Parameters & 0.234 & 0.678 & 0.456 & 0.568 & 0.7043 \\
\hline
\end{tabular}

Sometimes, some systems don't need adjust the five parameters of FOPID control online at same moment which will save the calculation time and make the overall system more ready to deal with the sudden disturbance. So, this study proposes three methods for self-tuning FOPID control. The first method considers $k_{p}, k_{i}$ and $k_{d}$ are constants while $\lambda$ and $\mu$ are varying. The second method considers $k_{p}, k_{i}$ and $k_{d}$ are varying while $\lambda$ and $\mu$ are constants. The third method tunes the five parameters of FOPID control online simultaneously.

\section{SIMULATION RESULTS}

This section presents the simulation results of the proposed different types of self-tuning FOPID control algorithms based on model reference. The first method, the adaptive mechanism will adjust the fractional integral and derivative parameters only while the other parameters are fixed. The second method, the fractional integral and derivative parameters are fixed while the other three parameters will be tuned using the adaptive mechanism. The third method, the five parameters of FOPID control will be adjusted simultaneously. Figure 3 demonstrates the Simulink diagram of the overall system with self-tuning FOPID based on optimal model reference technique.

A novel self-tuning fractional order PID control based on optimal model... (Mohamed. A. Shamseldin) 


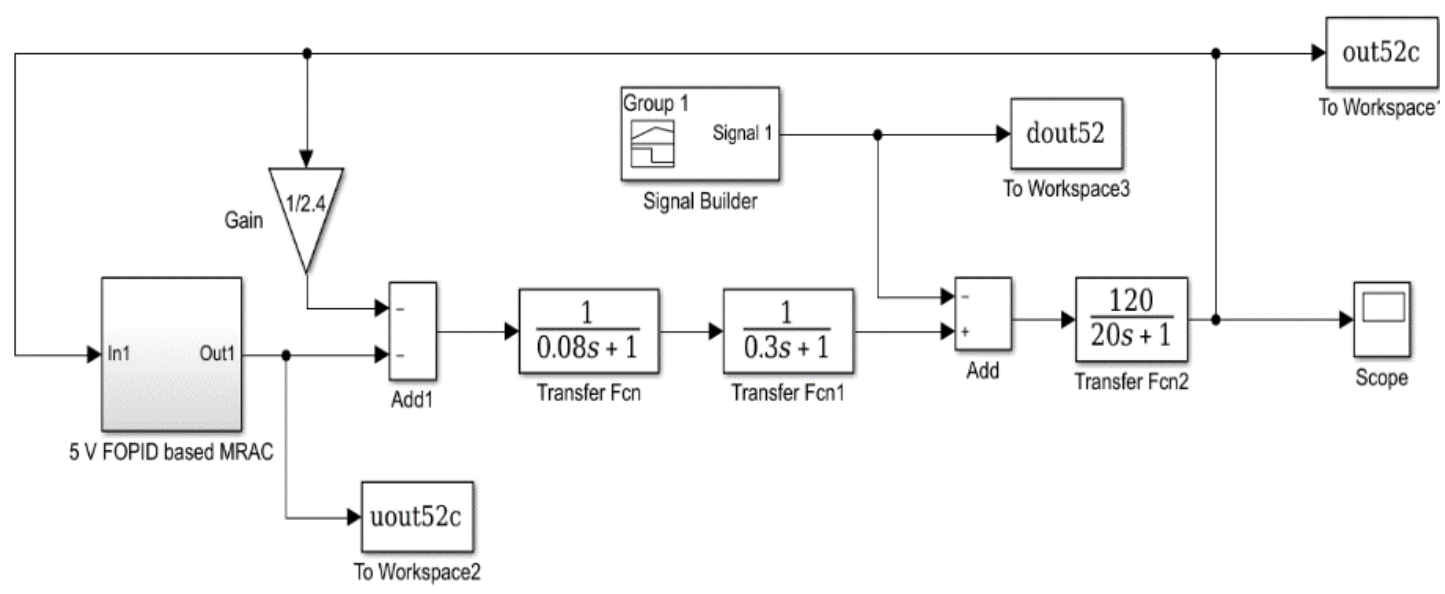

Figure 3. The simulink diagram of the overall system with self-tuning FOPID based on optimal model reference technique

The results are divided into two cases. The normal case where applied two types of the disturbers. These disturbers namely $\Delta \mathrm{Pd} 1$ as shown in Figure 4 and $\Delta \mathrm{Pd} 2$ as illustrated in Figure 5 . The parametric uncertainty case where to change the operating point to test the powerful of the proposed method against their counterparts

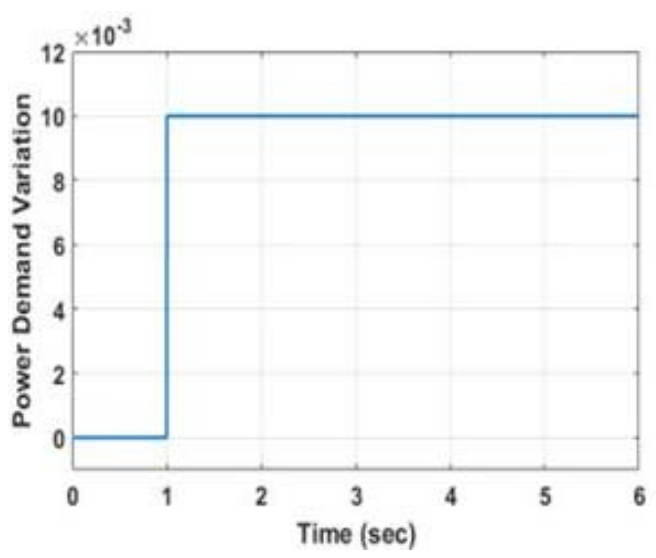

Figure 4. Power demand variation $(\Delta \mathrm{Pd} 1)$

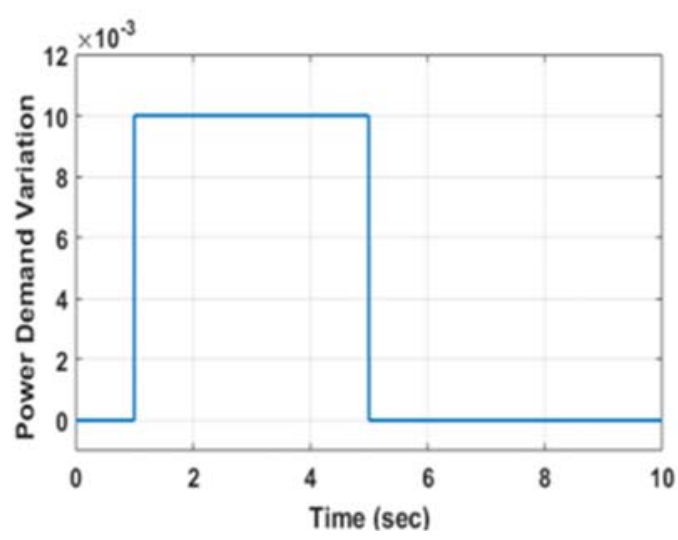

Figure 5. Power demand variation $(\Delta \mathrm{Pd} 2)$

\subsection{Normal case}

Two experiments were done at normal operating condition. The dynamic response of the system frequency $\Delta \mathrm{F}$ with the system driven by each type of the proposed controllers. In experiment number one where $\Delta \mathrm{Pd} 1$ is applied to the system. Figure 6 shows the time response of the system. Also, the output signal of each controller is shown in Figure 7. In experiment number two, Figure 8 demonstrates the time response of the system when $\Delta \mathrm{Pd} 2$ is applied to the system. Figure 9 presents the controller's outputs signals. 


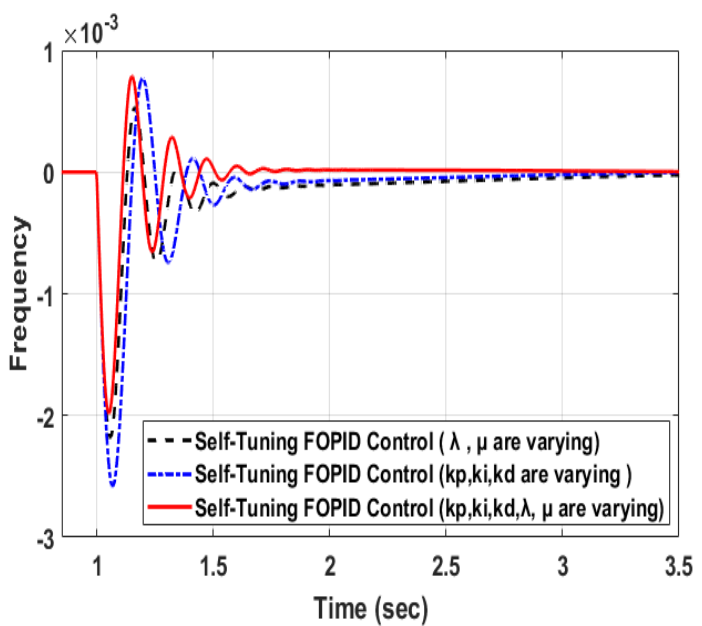

Figure 6. System dynamic responses at $\Delta \mathrm{Pd} 1$

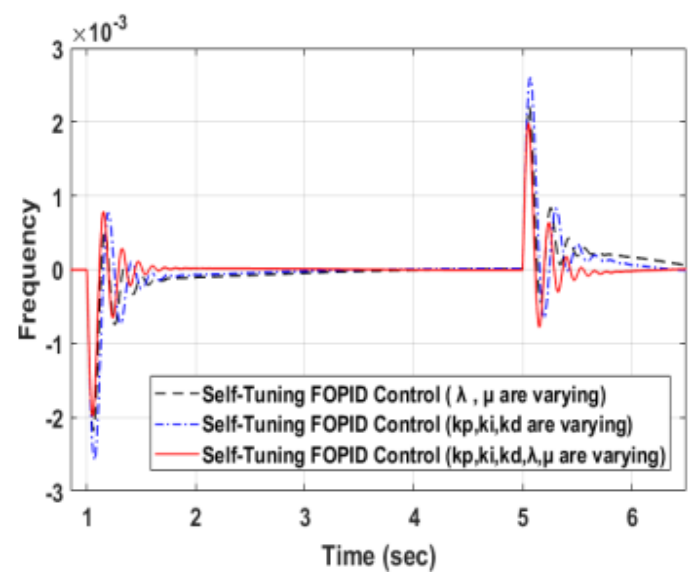

Figure 8. System dynamic responses at $\Delta \mathrm{Pd} 2$

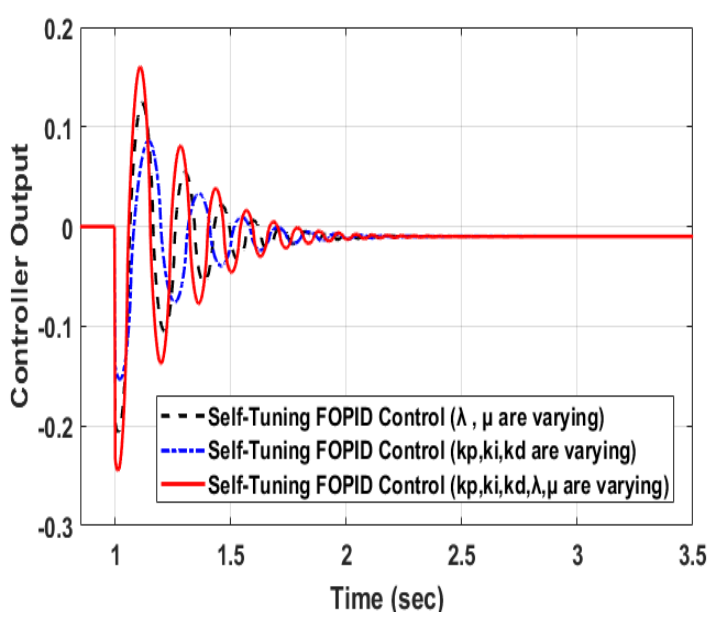

Figure 7. The controller's output signals at $\Delta \mathrm{Pd} 1$

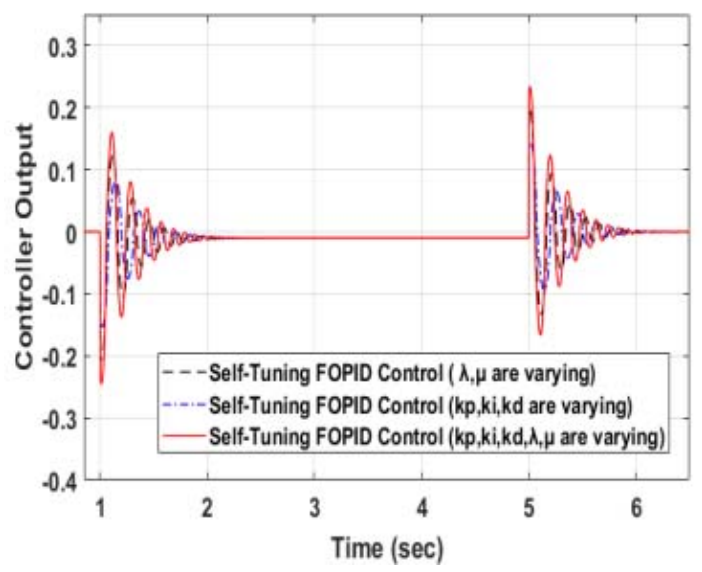

Figure 9. The controller's output signals at $\Delta \mathrm{Pd} 2$

It is clearly shown that The proposed third method of self-tuning FOPID controller gives battery performance with a smaller settling time and acceptable undershoot but with a relatively higher effort as shown in the response of its control output response $u$. also, the proposed third type of self-tuning FOPID controller recover to zero steady state error after a smaller time delay from the application of the two disturbance types.

\subsection{Parametric uncertainty case}

In this subsection, the parametric uncertainties of the power system have to be considered. According to load variation and power system configuration, the operation points of the system will be changed randomly during a daily cycle. The system parametric uncertainties are obtained by changing parameters by $50 \%$ from their normal values according to Table 3 and under the power demand variation $\Delta \mathrm{Pd} 2$. In this experiment, the power system responses at $\Delta \mathrm{Pd} 2$, including the effect of the parametric uncertainties is presented in Figure 10 and the controllers outputs are shown in Figure 11.

According to these results, it is clear that the proposed third method of self-tuning FOPID control shows the best response compared to other controllers. Also, the results approve the effectiveness and the ability of the proposed controller against the parametric uncertainties. Moreover, the undershoot at 1 second and overshoot at 5 second of third method of self-tuning FOPID control are significant small compared to other methods of self-tuning FOPID control. 
Table 3. Parametric Uncertainties

\begin{tabular}{cccc}
\hline Parameters & $\begin{array}{c}\text { Down } \\
(0-1) \mathrm{sec}\end{array}$ & $\begin{array}{c}\text { Normal } \\
(1-5) \mathrm{sec}\end{array}$ & $\begin{array}{c}\mathrm{Up} \\
(5-7) \mathrm{sec}\end{array}$ \\
\hline$k_{p}$ & 60 & 120 & 180 \\
$T_{p}$ & 10 & 20 & 30 \\
$\mathrm{R}$ & 1.2 & 2.4 & 3.6 \\
$T_{T}$ & 0.15 & 0.3 & 0.45 \\
$T_{G}$ & 0.04 & 0.08 & 0.12 \\
\hline
\end{tabular}

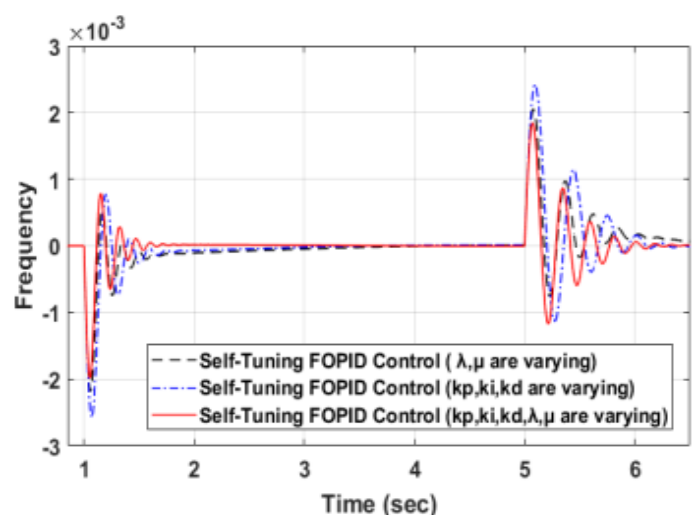

Figure 10. System dynamic responses at $\Delta \mathrm{Pd} 2$ and parametric uncertainties

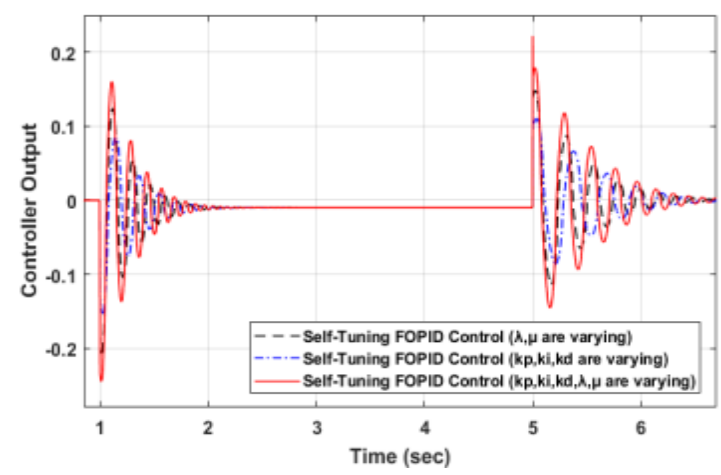

Figure 11. The controller's output signals at $\Delta \mathrm{Pd} 2$ and parametric uncertainties

\section{CONCLUSION}

A novel technique has been developed to tune the fractional order PID (FOPID) control online based on optimal Model Reference Adaptive Control (MRAC) for power system load frequency control as a case study. This work investigates the robustness of the proposed technique by applied different types of disturbances and parametric uncertainty. There are three methods of self-tuning FOPID control have been implemented. The first method, the three integer parameters (proportional, integral and derivative gains) are constant while the fractional integral and derivative gains are variable. The second method, the fractional integral and derivative gains are constant while the three integer parameters (proportional, integral and derivative gains) are variable. The third method, the all five parameters are variable and change continuously online. The simulation results demonstrate that the third method of self-tuning FOPID control can absorb the sudden disturbance faster than other techniques. Also, it can suppress the system uncertainty in a small time compared to other control techniques.

\section{REFERENCES}

[1] A. Scientiarum, "dSPACE real time implementation of fuzzy PID position controller for vertical rotating single link arm robot using four-quadrant BLDC drive," pp. 301-311, 2017.

[2] R. Madiouni, "Robust PID Controller Design based on Multi-Objective Particle Swarm Optimization Approach," in ICEMIS2017, pp. 1-7, 2017.

[3] P. Perz, I. Malujda, D. Wilczy, and P. Tarkowski, "Methods of controlling a hybrid positioning system using LabVIEW,” Procedia Eng., vol. 177, pp. 339-346, 2017.

[4] B. Zhang, G. Cheng, and J. Hu, "An Expanded Proximate Time-optimal Servo Controller Design for Fast Set-point Motion," Proc. 35th Chinese Control Conf. July, no. 2, pp. 4465-4470, 2016.

[5] M. Omar, M. A. Ebrahim, A. M, and F. Bendary, "Tuning of PID Controller for Load Frequency Control Problem via Harmony Search Algorithm,” Indones. J. Electr. Eng. Comput. Sci., vol. 1, no. 2, pp. 255-263, 2016.

[6] D. Vanitha and M. Rathinakumar, "Fractional Order PID Controlled PV Buck Boost Converter with Coupled Inductor," Int. J. Power Electron. Drive Syst., vol. 8, no. 3, pp. 1401-1407, 2017.

[7] M. A. A. Ghany, M. A. Shamseldin, and A. M. A. Ghany, "A Novel Fuzzy Self Tuning Technique of Single Neuron PID Controller for Brushless DC Motor," International Journal of Power Electronics and Drive System (IJPEDS), vol. 8, no. 4, pp. 1705-1713, 2017.

[8] M. A. Shamseldin, A. A. El-samahy, and A. M. A. Ghany, "Different Techniques of Self-Tuning FOPID Control for Brushless DC Motor," in MEPCON, 2016. 
[9] P. Kumar and S. Narayan, "Optimal design of robust FOPID for the aircraft pitch control system using multiobjective GA,” 2016 IEEE Students’ Conf. Electr. Electron. Comput. Sci., pp. 1-6, 2016.

[10] D. Tiwari, N. Pachauri, A. Rani, and V. Singh, "Fractional order PID (FOPID) controller based temperature control of bioreactor," Int. Conf. Electr. Electron. Optim. Tech. ICEEOT 2016, pp. 2968-2973, 2016.

[11] M. A. K. El-shafei, M. I. El-hawwary, and H. M. Emara, "Implementation of Fractional - Order PID Controller in an Industrial Distributed Control System," in 2017 14th International Multi-Conference on Systems, Signals \& Devices (SSD) Implementation, no. 3, pp. 713-718, 2017.

[12] I. Masngut, G. Nugraha, P. Pratama, A. I. Cahyadi, S. Herdjunanto, J. Fisher, and J. Pakpahan, "Design of Fractional-Order Proportional-Integral-Derivative Controller: Hardware Realization," in 2018 International Conference on Information and Communications Technology (ICOIACT) Design, no. 3, pp. 656-660, 2018.

[13] M. A. Shamseldin and A. A. El-samahy, "Speed Control of BLDC Motor By Using PID Control and Self-tuning Fuzzy PID controller," 2014.

[14] M. Irfan, M. Effendy, N. Alif, S. Lailis, I. Pakaya, and A. Faruq, "Performance Comparison of Fuzzy Logic and Proportional-integral for an Electronic Load Controller," International Journal of Power Electronics and Drive System (IJPEDS), vol. 8, no. 3, pp. 1176-1183, 2017.

[15] X. Gao, W. Zhao, and J. Huang, "On-line Parameter Identification and Control Parameters Optimization of Robot Joints with Unknown Load Torque," no. Icsai, pp. 61-66, 2016.

[16] D. V. L. N. Sastry and M. S. R. Naidu, "An Implementation of Different Non Linear PID Controllers on a Single Tank level Control using Matlab,” Int. J. Comput. Appl., vol. 54, no. 1, pp. 6-8, 2012.

[17] S. Slama, A. Errachdi, M. Benrejeb, A. R. B. F. Neural, and N. Identification, "Model Reference Adaptive Control for MIMO Nonlinear Systems Using RBF Neural Networks," in 2018 International Conference on Advanced Systems and Electric Technologies (IC_ASET), pp. 346-351, 2018.

[18] L. Zhu, X. Shi, Z. Chen, H. Zhang, and C. Xiong, "Adaptive Servomechanism of Pneumatic Muscle Actuators With Uncertainties," IEEE Trans. Ind. Electron., vol. 64, no. 4, pp. 3329-3337, 2017.

[19] M. Omar, "Harmony Search Based Fractional Order PID for Load Frequency Control."

[20] M. Omar, A. M. A. Ghany, and F. Bendary, "Harmony Search based PID for Multi Area Load Frequency Control Including Boiler Dynamics and Nonlinearities," WSEAS Trans. CIRCUITS Syst., vol. 14, pp. 407-414, 2015.

[21] K. Hossain and S. M. R. Islam, "Model Reference Adaptive Control for Surface Permanent Magnet Synchronous Machine," no. December, pp. 7-9, 2017.

[22] S. Wen, T. Wang, Z. Ma, and X. Li, "Dynamics Modeling and Fuzzy PD Control of Humanoid Arm," in Proceedings of the 36th Chinese Control Conference, no. 3, pp. 616-621, 2017. 